\title{
La formation initiale différée
}

\section{Daniel Bancel et Jocelyne Nosenzo}

\section{(2) OpenEdition}

Journals

Édition électronique

URL : http://journals.openedition.org/ries/3044

DOI : 10.4000/ries.3044

ISSN : 2261-4265

\section{Éditeur}

Centre international d'études pédagogiques

\section{Édition imprimée}

Date de publication : 1 décembre 1997

Pagination : 95-100

ISSN : 1254-4590

\section{Référence électronique}

Daniel Bancel et Jocelyne Nosenzo, «La formation initiale différée », Revue internationale d'éducation de Sèvres [En ligne], 16 | Décembre 1997, mis en ligne le 07 juin 2013, consulté le 03 mai 2019. URL http://journals.openedition.org/ries/3044; DOI : 10.4000/ries.3044

Ce document a été généré automatiquement le 3 mai 2019.

(c) Tous droits réservés 


\title{
La formation initiale différée
}

\author{
Daniel Bancel et Jocelyne Nosenzo
}

1 Cet article décrit une démarche privilégiant le pragmatisme et l'efficacité qui a fait son chemin et a fini par se concrétiser grâce à la volonté des partenaires (conseil régional Rhône-Alpes, conseil régional de l'ordre des experts comptables de Lyon, AGEFOS PME Rhône-Alpes, académie de Lyon). A posteriori, en nous retournant, nous essayerons de montrer que cette expérience est pleine d'enseignement pour « la formation tout au long de la vie».

\section{Une démarche partenariale}

2 Au départ, il y a la rencontre de deux préoccupations, l'une est liée à l'orientation des bacheliers professionnels, l'autre est liée à la gestion prévisionnelle des compétences pour une profession, celle des experts-comptables.

3 Côté orientation, il s'agit d'accroître le nombre de jeunes qui sortent du système scolaire avec le baccalauréat (essentiellement le baccalauréat professionnel, éventuellement le baccalauréat technologique) pour entrer dans une entreprise et y mettre en œuvre dans une première expérience professionnelle, les qualifications et compétences certifiées par le diplôme qu'ils viennent d'acquérir.

4 Aujourd'hui, le projet du plus grand nombre de ces jeunes bacheliers (et de leurs parents) consiste dans une poursuite d'études qui, souvent, ne sera interrompue que par l'échec. Alors même que les études prospectives affichent que pour un pourcentage important, les premiers emplois continueront à faire appel à des fonctions ou qualifications de niveau IV 1 .

5 Cette demande sociale de poursuite de scolarité se nourrit souvent de l'aspiration à atteindre, à terme, au sein de l'entreprise un niveau d'emploi correspondant à des fonctions d'encadrement, fonctions pour lesquelles le niveau de formation exigé est difficile à acquérir en dehors de la formation initiale. Certains jeunes répugnent à 
intégrer le monde du travail par peur de ne pas avoir un capital de diplômes suffisant pour l'ensemble de leur carrière professionnelle.

6 Savoir que l'on peut non seulement revenir en formation, mais aussi valider les acquis de l'expérience professionnelle tout en progressant au sein de l'entreprise, est, évidemment, porteur d'espoir : cela prouve que les jeux ne sont pas faits une fois pour toutes.

7 Coté entreprise, il s'agit d'anticiper les besoins de qualification, de préparer aussi les futures évolutions, de disposer d'un outil de gestion prévisionnelle des compétences. Dans un secteur en pleine évolution, les cabinets d'expertise comptable délaissent les activités purement comptables que leurs clients effectuent de plus en plus eux-mêmes avec des logiciels et s'orientent vers le conseil.

Pour faire face à cette évolution, une première solution, la plus évidente, consiste à recruter au niveau BTS au minimum. Se pose alors, à terme, le problème des salariés embauchés dans les dix dernières années avec un niveau de formation équivalent au baccalauréat et qui occupent des emplois d'assistants comptables. Ces jeunes ne peuvent envisager une évolution dans la profession qu'en préparant, en promotion sociale, des diplômes (DPECF, DECF, DESCF²) qui ne s'obtiennent bien souvent, dans ces conditions, qu'à l'issue de plusieurs années d'études et qu'au prix d'un dur labeur et de sacrifices importants dans leur vie familiale. Beaucoup de ceux qui se lancent dans cette aventure abandonnent par découragement, même s'ils perçoivent la difficulté d'évoluer sans diplôme.

Une seconde solution, le dispositif de "formation initiale différée » se propose de réaliser simultanément l'évolution des compétences et des fonctions au sein de l'entreprise et l'acquisition d'un diplôme. Une telle solution doit donc valoriser au maximum la capacité formatrice du poste de travail. Elle doit intégrer dans le plan de formation les contraintes comme les souplesses de l'activité au sein de l'entreprise.

10 Permettre aux jeunes sans quitter leur entreprise de bénéficier d'une formation individualisée qui tienne compte des acquis de leur expérience professionnelle et soit adaptée aux nécessités (notamment en termes horaires) et aux contraintes de l'entreprise : voilà quel était le cadre dans lequel devait se construire la formation initiale différée.

11 Les principes fondateurs du projet ont remporté la conviction des partenaires. Un partenariat institutionnel s'est organisé et a conduit à la signature au printemps 1993, d'une convention entre le rectorat, l'ordre des experts-comptables, le conseil régional et l'AGEFOS-PME.

12 Le premier dispositif, expérimental, fut implanté au lycée Albert Camus de Rillieux et au GRETA tertiaire de Lyon, en particulier avec l'appui du centre permanent informatique (dispositif spécialisé dans la formation individualisée des adultes).

\section{Le dispositif de formation}

13 L'origine même du projet a conduit à repérer et à valider les acquis de l'expérience professionnelle en s'appuyant sur la loi de 1992 sur la validation des acquis de l'expérience professionnelle. Il peut être noté que cette formation a constitué un premier terrain d'expérience dans l'académie pour l'utilisation de cette nouvelle procédure. 

réduire de trois cents heures le temps de formation. Outre le ciblage de ces acquis professionnels à valider, l'étude de l'activité professionnelle de chaque salarié, de son contexte, de son évolution possible dans la structure, conduite avec la collaboration active des salariés, de leur tuteur, a favorisé le repérage des apprentissages qu'il était possible d'intégrer à l'activité professionnelle et la construction d'un plan individuel de formation par alternance. Chaque salarié a pu ainsi réaliser trois cents heures au moins d'apprentissage au poste de travail accompagné par un tuteur. Les formateurs de chacun des domaines concernés ont procédé, en lien étroit avec les tuteurs, à l'évaluation de ces apprentissages dans l'entreprise tout au long du parcours de formation. Les formateurs, venant de la formation continue (GRETA ${ }^{3}$ ) comme ceux venant de la formation initiale (lycée) ont assuré cette évaluation au poste de travail (et, ensuite, le suivi individualisé des salariés) avec conviction et dynamisme dans une relation très riche avec les cabinets d'expertise-comptable. individuel de formation au centre permanent. Un dispositif flexible dit «à guichets ouverts » fut organisé au centre permanent. Adapté aux fluctuations de la charge de travail dans le secteur professionnel, il a permis de proposer trois mille heures de formation durant dix-huit mois pour faciliter la réalisation de parcours individualisés dont la durée n'excédait pas neuf cents heures. À des périodes de travail intensif (période des bilans comptables, par exemple) a correspondu une offre minimale du centre permanent; à des périodes de "moindre activité professionnelle" a correspondu une offre de formation intensive. Le centre permanent communiquait tous les deux mois aux cabinets d'expertise-comptable concernés l'offre de formation. Chaque salarié convenait alors avec le tuteur, en fonction des impératifs de l'activité, des plages de formation à retenir et envoyait sa demande par télécopie. On réalise sans difficulté que cela ne va pas sans aléas. La gestion n'est pas simple mais ce système "à la carte » a été, après une période d'adaptation, parfaitement maîtrisé et fort apprécié des salariés et des employeurs. d'acquisition et les modes d'apprentissages. En fonction des acquis identifiés au début de la formation, les durées de formation ont pu varier du simple au triple. Par exemple, en mathématiques, de 50 à 150 heures.

17 Il serait probablement intéressant de faire une approche cognitive de cette expérience. Certains ont adopté une démarche inductive partant de leur expérience concrète du traitement des dossiers clients dans le cabinet, ils ont ensuite formalisé avec l'aide du tuteur une approche plus théorique. D'autres ont adopté une démarche déductive partant de l'étude théorique pour ensuite se pencher sur les cas concrets des dossiers clients du cabinet.

\section{Le montage financier}

Il est à la hauteur du projet et à l'image du partenariat. Il traduit la convergence d'intérêts existant à propos de cette formation et la volonté des partenaires de rassembler des moyens suffisants pour la faire vivre et la mener à bien.

Revue internationale d'éducation de Sèvres, 16 | 2013 
19 S'agissant de jeunes salariés qui conservaient leur emploi tout en réalisant leur formation, il convient de souligner que le coût des salaires et des charges, pendant leur temps d'absence au poste, a été imputé sur « le plan de formation de l'entreprise ».

Compte tenu de la part de formation qui a pu être intégrée au poste de travail dans l'entreprise, compte tenu aussi de la part de formation que chaque salarié a réalisé sur son temps personnel, le temps d'absence au poste n'a pas excédé 700 heures durant ces deux années de formation.

On peut donc calculer le coût des salaires et des charges relatifs à la formation prise sur le temps de travail. Ce coût a incombé à AGEFOS PME, organisme collecteur de fonds de formation et aux cabinets comptables. AGEFOS PME a concrétisé sa volonté de contribuer de façon tout à fait significative à la prise en charge de la formation des salariés des plus petits cabinets, en remboursant $80 \%$ au moins du coût des salaires et charges relatif au temps d'absence des salariés lorsqu'il s'agissait de cabinets de moins de dix salariés. Pour les cabinets de dix salariés, la prise en charge a été de $50 \%$ et plafonnée, quelle que soit la taille du cabinet, à 70000 francs.

Concernant le coût de fonctionnement pédagogique, le budget présenté aux partenaires s'élevait à 88000 francs par stagiaire. Outre les frais de personnels enseignants, frais pédagogiques, frais de déplacements, frais de locaux, frais généraux, ce budget affichait des frais spécifiques liés à l'ingénierie de cette première expérience.

Le conseil régional Rhône-Alpes a concrétisé sa volonté d'encourager ce type de formation en accordant une subvention spécifique pour l'ingénierie. Celle-ci a permis d'abaisser le coût du fonctionnement pédagogique à 82000 francs par salarié. Le conseil régional a proposé par ailleurs de subventionner les parcours de formation au même titre et au même taux que les formations d'apprentis, prenant ainsi à sa charge $28 \%$ du coût pédagogique restant (23000 francs par salarié).

À projet nouveau, moyens nouveaux. Considérant que ce type de formation relevait à la fois de la formation initiale et de la formation continue, le rectorat a accordé un poste et demi de formateur pour la durée de cette formation (deux années scolaires). Cette contribution a représenté $40 \%$ du coût pédagogique. Restaient à la charge des cabinets et d'AGEFOS PME environ 25000 francs par salarié. Afin de faciliter la formation dans les petits cabinets, AGEFOS PME a souhaité rembourser $80 \%$ au moins de cette somme aux cabinets comptant moins de dix salariés et $50 \%$ au moins à ceux en comptant plus de dix, réduisant ainsi considérablement leur contribution financière.

Si l'on met à part le coût généré par l'ingénierie de cette première expérience, on constate que le coût de cette formation n'a rien d'excessif et est comparable à celui de la formation initiale délivrée sur deux années.

\section{Évaluation et réflexions}

Le premier critère d'évaluation a été le taux de réussite au diplôme : il est classique et, de plus, s'est révélé déterminant pour l'évolution du salarié et du cabinet. Ce label "Éducation nationale » justifiait a posteriori les efforts consentis à titre individuel ou par solidarité pour les collègues du cabinet.

Entrés en formation en novembre 1993, 80 \% des salariés-stagiaires ont obtenu, en dixhuit mois, la totalité des unités constitutives du diplôme. Il faut préciser que l'essentiel 
des échecs partiels réside dans l'impossibilité pour certains salariés de " consommer » en dix-huit mois la totalité du crédit formation alloué sur le temps de travail ; certains échecs ne sont que momentanés, la réussite n'est qu'une question de temps puisqu'il s'agit de présenter les unités manquantes.

Les petites structures de quatre ou cinq salariés n'ont pu quelquefois s'organiser de manière à libérer suffisamment les salariés-stagiaires.

Les résultats de la première promotion montrent l'importance des facteurs autres que le niveau de formation : organisation, «professionnalisme » pour mener à bien son projet, solidarité des collègues du cabinet.

Le second critère d'évaluation a été l'évolution de l'activité des salariés. Le bilan est positif, l'évolution s'est majoritairement négociée au sein du cabinet, l'activité professionnelle évoluant vers des fonctions d'encadrement et vers le conseil juridique et économique aux clients, avec souvent des augmentations immédiates de salaire.

La mise en place de la formation initiale différée a mis en évidence des exigences fortes :

- admettre que l'on n'apprend pas qu'à l'école et que le travail lui-même est formateur, source d'apprentissages techniques, sociaux, humains. Ce principe déjà largement appliqué dans l'alternance suppose une petite mais véritable révolution dans les mentalités ;

- trouver dans les entreprises des emplois de base laissant suffisamment de place à l'expression de la compétence et de l'autonomie ;

- mettre en œuvre une organisation de travail permettant l'évolution des compétences dans un métier comme au sein du système hiérarchique d'encadrement.

L'esprit de partenariat et la mutualisation des ressources ont fait passer ce projet du stade de l'idée à celui de la réalité. Sans cette conviction partagée que désormais la vie sera faite d'allers et retours réguliers entre des situations de travail et de formation, chacune enrichissant l'autre, notre partenariat n'aurait été qu'une déclaration d'intention. Depuis cette première expérience, d'autres BTS peuvent être acquis en formation initiale différée : assistant de gestion PME/PMI, maintenance, productique, avec de nouveaux partenaires pour les professions.

Le temps n'est plus où l'on pouvait se contenter d'apprendre d'abord et de travailler ensuite, les jeux ne sont pas faits une fois pour toutes et il faudra au contraire, de plus en plus, tracer son propre chemin en ne misant pas uniquement sur le « tout école » mais en serpentant entre formation et action, entre épanouissement personnel et implication professionnelle. La formation initiale différée a des allures de nouvelle forme de promotion sociale.

\section{NOTES}

1. Niveaux I et II : diplômé de $2 \mathrm{e}$ et $3 \mathrm{e}$ cycle supérieur. Niveau III : bac + deux années d'études avec le diplôme de ce niveau. Niveau IV : niveau bac ou abandon avant le niveau III. Niveau V : niveau CAP/BEP ou abandon avant le niveau V. 
2. DPECF : diplôme préparatoire aux études comptables et financières. DECF : diplôme d'études comptables et financières. DESCF : diplôme d'études supérieures comptables et financières.

3. GRETA : Groupement d'établissements pour la formation continue.

\section{RÉSUMÉS}

La formation initiale différée est un exemple de dispositif favorisant la formation tout au long de la vie. Examinée du point de vue de la démarche, du coût et de l'impact, elle apparait bien comme en rupture avec la formation continue classique : elle ne s'effectue pas en plus du travail, ni pendant une interruption de carrière, ou une pause choisie. Mais elle se déroule sur le lieu de travail et permet de compléter, sans prendre la forme d'un stage, « une formation initiale non conduite à terme ».

INDEX

Index géographique : France

Mots-clés : validation des acquis de l'expérience, formation professionnelle, individualisation, relation école-entreprise

\section{AUTEURS}

\section{DANIEL BANCEL}

Recteur, académie de Lyon, France.

\section{JOCELYNE NOSENZO}

Conseiller en formation continue, académie de Lyon, France. 